\title{
A journey towards reducing colon cancer burden
}

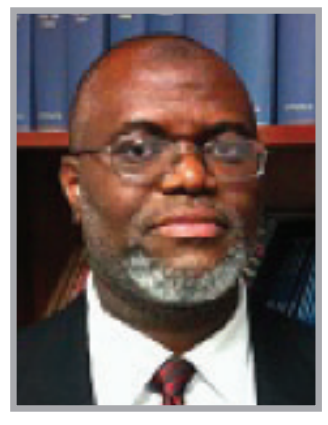

Adeyinka 0 Laiyemo* speaks to Natasha Galukande, Commissioning Editor: Dr Laiyemo is currently an Assistant Professor in the Division of Gastroenterology at the Howard University College of Medicine (Washington, DC, USA), and is heavily involved in studies focusing on racial disparity in colorectal cancers. He graduated from the University of Lagos (Nigeria) in 1990, before working as a General Medical Officer at two hospitals from 1990-1996. Dr Laiyemo had his medical internship, medical residency and gastroenterology fellowship at Howard University College of Medicine from 1998-2006. In 2005 he was a cancer prevention fellow at the National Cancer Institute (MD, USA) and in 2006 he graduated from Johns Hopkins University (MD, USA), majoring in epidemiology and biostatics. In recent years Dr Laiyemo has been part of the National Cancer Institute's Alpha Tocopherol Beta Carotene (ATBC) Cancer Prevention Trial Study Group, the Polyp Prevention Trial (PPT) Study Group, the Prostate, Lung, Colorectal and Ovarian Cancer Screening Trial (PLCO) Colon Subcommittee and the Health Information National Trends Survey. Laiyemo is a member of the Howard University Advisory Committee on Graduate Medical Education, the American Association for Cancer Research, the American College of Physicians, the American College of Gastroenterology and the Alpha Omega Medical Honor Society. He was recently awarded the 'Minority Scholar in Cancer Research Award' at the 102nd American Association for Cancer Research Meeting in Orlando ( $F L, U S A)$.

Q What sparked your interest in colorectal cancer?

Well that's a very interesting question. I definitely did not start my career thinking I would be doing cancer research, not at all. What actually happened was some time in December 2000, when I was doing my oncology rotation, I was seeing a lot of people with cancer. Some of them were quite young, including a particular young lady, who in her mid-20s, was dying of metastatic breast cancer. Because of the many young people with cancer I was not enjoying my oncology rotation. It was obvious to my attending doctor and he told me: "Dr Laiyemo, let me know when you think you are only taking care of people who are going to die anyway so I can take you off my rotation. Maybe you should actually consider doing something about it."

The following month, which was my scheduled period of vacation, I decided to volunteer in the cancer center. In my own little world this was my way of doing something about it. I worked with one of our researchers looking at microsatellite instability in colon cancer, which was interesting. We eventually presented our findings at the Digestive Diseases Week (May 2003, FL, USA) [1]. My interest in research was first sparked.

By the time I finished internal medicine and I did a gastroenterology fellowship the goal was 'I need to get into research now so

*Division of Gastroenterology, Department of Medicine, Howard University College of Medicine, Washington, DC, USA; adeyinka.laiyemo@howard.edu

\section{News \& Views}

News

Journal Watch

Interview

Conference Scenes 
I can do something, no matter how small it may be, because it will make me feel better'. This thought sparked my interest a lot more and I applied for a position at the Cancer Prevention Fellowship Program (CPFP) in the National Cancer Institute (MD, USA). Fortunately I was among the successful candidates and I went on to obtain a masters degree from Johns Hopkins University (MD, USA). Since then I've been trying to contribute towards preventing colon cancer, which is really my area of interest.

Q What do you feel is the biggest challenge facing the field today?

I think there are still so many things that we don't know. That itself is the biggest challenge and the driving force for the excitement surrounding the field. One of the things I would love to know as a person - if I had a genie in a bottle, shook it and was asked to make a wish - is can we point to someone who will develop colon cancer in the future? I think that would be great to be able to identify people at risk, carry out a colonoscopy and tell who will or will not develop polyps, and whose polyp will become cancerous. It would be great to be able to understand why someone will have cancer in the rectum or the sigmoid colon as opposed to the descending colon. The biggest challenge to the field is actually the lack of knowledge in some areas.

I think we are making progress, but for someone like me whose interest is to eliminate all cancer, I personally feel that we are running out of time, at least in my lifetime. There is currently a lot of interest in the field of cancer prevention and a lot of people are donating money for research and investing time and resources that are helping us to move forward. All of these are positive steps but we are still far from the ideal situation of eliminating cancer altogether.

Q How did you initially get involved in studying racial differences in colorectal cancer? How has this field developed in recent years?

That's a great question. I started out trying to prevent colon cancer in general and then in the process of studying and being more involved in the field I realized that even though colon cancer may be a really bad disease that affects lots of people, some people are even more affected by it than others. That knowledge led to us identifying people that are at an increased risk of sporadic types of cancer. Sporadic cancer just occurs and we can't say if there is a particular gene or defect that is causing it. Then I realized that there are some groups that disproportionately have a higher incidence of it, which got me interested in seeing if we could find a way to at least reduce cancer burden in those groups. That would be something worthwhile in terms of 'if you cannot cure all cancer then at least start from a small group of people and gradually expand'.

I was fortunate enough to be at the NIH (MD, USA) at the time, which had ongoing clinical trials and gave me the opportunity to study this at the beginning. I found it really interesting to study and my mentors assisted and guided me in terms of understanding the field and the core issues.

In recent times there has been increasing interest in reducing disparity by race because African-Americans are more likely to develop colon cancer and die from it than any other race/ethnicity. A lot of interest has been focused on what we can do to reduce this. The field is developing and a lot interventions are being evaluated; hopefully in the next few years we should be able to say that we have discovered, what we need to do to stop this and are able to implement these changes, that would be a real dream come true.

\section{Q Who is responsible for making} \& implementing changes that will benefit the public in the future?

This is a question of two parts. First, someone who is able to identify a change that needs to be made and is in a good position to implement this. There are some changes that would be great but an individual is unable to carry out. Therefore, these changes would have to be done by the community together or by the government. For example, we don't have healthcare coverage for everybody in the USA. So if somebody has an emergency you can go the emergency room but you can't go to the emergency room and say 'I think I am at high risk for colon cancer, can you 
please check me out?' The people at the emergency room will tell you to go to your primary doctor but if you don't have insurance then you won't have a primary doctor. Therefore, a policy change like that is essential, which is why it is good that the USA is moving towards a system where everybody will have access to insurance, and screening is part of that.

As good as it is that the government can make a change that means everybody gets access to healthcare insurance, even if an individual has insurance it does not mean that he will use it, which is where the second part, individual responsibility, comes in. There are many people that have insurance coverage, primary care doctors and the hospital may even be across the road, but they will never go in. A lot has to be done at an individual level in terms of education and knowing the fact that we have our destiny in our hands in terms of how we approach preventive healthcare because many people wait until they are sick. The concept of prevention versus going to your doctor once you are sick needs a lot of work, and in that regard we need to let people understand the changes that will benefit the public in the future have to be done in collaboration with the government, the public sector, the private sector and of course, the individual. Individuals need to be ready to make these changes.

Q There are several videos in which you discuss important issues surrounding colorectal cancer available on the internet. What do you think the impact of these videos are \& who is your target audience?

In terms of what the impacts of those videos are, honestly I don't know. I've seen many people that have watched the videos and they have engaged me in discussions. The target audience is everybody as everybody can get colorectal cancer.

At the beginning of lectures at places like shelters, community centers, churches, mosques and old people's homes, I always tell them "well, the people who need to worry about colon cancer will be everybody except one group," and then usually I like to ask them questions in that regard such as "who would be in the group of people that do not need to worry about colon cancer?" Many times people try and guess the answer of who would be included in this group, which of course is "anybody that does not have a colon." As long as you have a colon there is a chance you can get colon cancer.

What I hope to achieve by the videos is making sure that everybody with a colon knows that there is a chance of them getting colon cancer so they can take the necessary steps to prevent the disease.

One of the fantastic aspects of the internet is that people can always watch it on their own, at their own leisure, with no time limit. In between people watching cat videos, they can also watch videos on how to prevent colon cancer. Instead of sports they can watch how to stay healthy and prevent diseases.

Q What is your involvement in teaching young health professionals \& what do you enjoy most about this role?

I am going to answer this question philosophically. As a young man I almost could not fathom how my teachers in elementary school were always so happy because we were just kids, and kids can behave badly. But they see us graduating from school and they are happy to see us make progress. When you go back to your elementary school or high school the teachers that once knew you are still always so happy to see you and are interested to hear how things are with you. In many cases you have moved up higher than the teachers on the 'food chain' and are now more accomplished than them, and yet they are still always so happy, which is something that I now understand.

It is their joy that they are able to make meaningful contributions to those who will advance things in the future. I realized this when I attended the graduation ceremony of our medical students. I could remember the first day when they walked in and they couldn't examine the abdomen properly. You give them assignments and they roll their eyeballs, but by the time they finish their training and are in their long white coats they are happy and you feel very happy. As a teacher you turn to them and say to yourself "guys, now it is your turn to ensure you 
keep the flag flying". It is such a great joy to know that you are passing on the baton to continue the race for the good of mankind. It is something difficult to describe but I know that I enjoyed every minute of it.

Teaching young professionals is a large part of what I do because at the end of the day there is no benefit if you have a whole lot of knowledge and then the knowledge is gone at the end when you die. Passing on knowledge is the best part of being a doctor and the best doctor is a teacher. It allows people to advance, and as a doctor you do your best to advance it, even if it is only by a very small amount. Someone else can then advance it further as we have to keep moving forward.

\section{Q As a member of the Howard} University Advisory Committee on Graduate Medical Education, the American Association for Cancer Research, the American College of Physicians, the American College of Gastroenterology \& the Alpha Omega Medical Honor Society, how do you manage your time between these roles? Somebody once said the following quote that I totally agree with "if you enjoy what you do, you won't think you are working." It is an investment of time to belong to and be actively involved in lots of associations and the amount of time that each requires varies quite a lot. If you are only doing one job that you hate then it feels the whole world is crashing in on you; however, if you are doing multiple things that are fun, then you will enjoy it and you will not feel busy.

Q What do you think is the biggest achievement of your career so far?

Teaching the next generation and carrying out research that will provide us with knowledge that can benefit mankind. I still have a lot left to achieve in that regard though, but I feel proud of what I have achieved so far and it makes me happy.

\section{Q What are the current hot topics in colorectal cancer research?}

That depends on the individual perception but I would say that interval cancer is a very important topic at this point in time, when the patient comes back with colon cancer after you have performed screening. We are currently unsure if the quality of the examinations is the issue, the operator, the endoscopist or if the biology of the cancer is different; there are so many factors to this that we do not know and therefore it's a hot topic.

The other hot topic is the ability to predict cancer, anything that helps us to understand cancer better in terms of what we need to do, either to prevent the disease or to ensure that the disease does not come back. Even though we have effective therapies these therapies are almost never $100 \%$. If we can prevent cancer, then we don't have to worry about what it takes to cure the disease. However, we may not be able to prevent some of these cancers; therefore, we need to work on what to do when the patient has the disease.

Q What progress do you think will be made in colorectal cancer treatment in the next 10 years?

The first thing I will hope for in the next 10 years is a prevention or cure for all colorectal cancer! I am an optimist though, so if we don't achieve that then the progress that I'm hoping to see is personalized care for each individual. I am hoping that we will be able to identify the biology, find the particular target therapy that we could apply, find the most specific therapy that will lead to an improved quality of life, reduced chemotherapeutic toxicity and a reduction in the risk of recurrence. These are changes that, being an optimist, I think will be made in the next 10 years. I tell trainees and colleagues that they should help, by trying and tracking their way back to academia and research to ensure that more people are working on improving the situation with colorectal cancer. We are more likely to get answers quicker if more people are invested in it.

I would love to see a world where everybody understands that we all have to work together to reduce the incidence of colorectal cancer. There are many communities where people do not talk about cancer at all, let alone colorectal cancer. There are many communities where the people do not want to get checked for 
colon cancer and there are many people that have different ideas and misconceptions about colonoscopy as a screening technique. I am looking for a world where people feel comfortable to discuss these issues with their families, which especially needs to happen to combat colorectal cancer race disparity. In African-American communities we generally don't want to talk about colon cancer, so anything that will facilitate these conversations would be a wonderful and welcome development. In terms of when somebody gets a colon check they should tell their family members and encourage them also to get checked. So this will be something really important to move forward. Finally, it is important to ensure that people without health insurance get it, and those that have it use it to make sure they are getting checked for this potentially preventable disease.

\section{Disclaimer}

The opinions expressed in this interview are those of the interviewee and do not necessarily reflect the views of Future Medicine Ltd.

\section{Financial \& competing interests}

\section{disclosure}

AO Laiyemo has no relevant affiliations or financial involvement with any organization or entity with a financial interest in or financial conflict with the subject matter or materials discussed in the manuscript. This includes employment, consultancies, honoraria, stock ownership or options, expert testimony, grants or patents received or pending, or royalties.

No writing assistance was utilized in the production of this manuscript.

\section{Reference}

1 Hassan A, Smoot DT, Rahmanian M et al. Cliniopathological features and MSI in colorectal cancers from African Americans. Gastroenterology 124(4), A237-A238 (2003). 